\title{
Exercise Alters Mineral and Matrix Composition in the Absence of Adding New Bone
}

\author{
David H. Kohn ${ }^{a, b}$ Nadder D. Sahar ${ }^{b} \quad J o s e p h ~ M$. Wallace $^{b} \quad K_{\text {Kurtulus Golcuk }}^{c}$ \\ Michael D. Morris ${ }^{c}$ \\ Departments of a Biologic and Materials Sciences, ${ }^{b}$ Biomedical Engineering and ${ }^{c}$ Chemistry, University of Michigan, \\ Ann Arbor, Mich., USA
}

\section{Key Words}

Mechanical loading $\cdot$ Mechanical properties $\cdot$ Mineral

Collagen • Raman spectroscopy

\begin{abstract}
The mechanical properties of bone are dictated by its amount, distribution and 'quality'. The composition of the mineral and matrix phases is integral to defining 'bone quality'. Exercise can potentially increase resistance to fracture, yet the effects of exercise on skeletal fragility, and how alterations in fragility are modulated by the amount, distribution and composition of bone, are unknown. In this investigation, the effects of exercise on the size, composition, mechanical properties and damage resistance of bones from mice of various ages, background strains and genetic makeup were assessed, as a means of testing the hypothesis that mechanical loading can improve skeletal fragility via compositional alterations. C57BL/6 mice (4-month-old males) ran on a treadmill for 21 days. Tibiae from exercised and control mice were analyzed for cross-sectional geometry, mechanical properties, microdamage and composition. Exercise significantly increased strength without increasing cross-sectional properties, suggesting that mechanical stimulation led to changes in the bone matrix, and these changes led to the improvements in mechanical properties. Consistent with this interpretation, the mineral/matrix ratio was
\end{abstract}

significantly increased in exercised bones. The number of fatigue-induced microcracks was significantly lower in exercised bones, providing evidence that exercise modulates fatigue resistance. The ratio of nonreducible/reducible crosslinks mirrored the damage data. Similar trends (exercise induced increases in mechanical properties without increases in cross-sectional properties, but with compositional changes) were also observed in 2-month-old biglycan-deficient and wild-type mice bred on a C57BL/6x129 genetic background.

Copyright $\odot 2008$ S. Karger AG, Basel

\section{Introduction}

Each year, an estimated 1.5 million Americans suffer an age-related fracture, resulting in direct care expenditures of USD 18 billion/year [United States Department

\section{Abbreviations used in this paper}

ANOVA analysis of variance

ECM extracellular matrix

PMMA polymethylmethacrylate

SEM standard error of the mean

TGF- $\beta$ transforming growth factor- $\beta$

\section{KARGER}

๑ 2008 S. Karger AG, Basel

Fax +4161306 1234

E-Mail karger@karger.ch

www.karger.com
Accessible online at: www.karger.com/cto
Dr. David H. Kohn

Departments of Biologic and Materials Sciences and Biomedical Engineering University of Michigan, 1011 N. University Ave

Ann Arbor, MI 48109-1078 (USA)

Tel. +1 734764 2206, Fax +1 734647 2110, E-Mail dhkohn@umich.edu 
of Health and Human Services, 2004]. Bone mass is the most common diagnostic used to assess skeletal fragility, yet it only predicts half of all fractures. Bone fracture and, more generally, bone mechanical properties are dictated not only by the amount of bone, but also the distribution and 'quality' of the tissue [Burr et al., 1997]. One set of metrics gaining importance in defining bone quality is the composition of the mineral and matrix phases [Timlin et al., 2000; Carden et al., 2003].

Exercise can potentially increase resistance to skeletal fragility, and it is commonly thought that mechanical stimulation exerts its influence through structural adaptation and the accrual of bone mass [Carter et al., 1996]. Rodent models of exercise have shown a link between mechanical loading of bone and increased bone formation, resulting in increased bone mass and cross-sectional geometric properties, and maintenance or increase in mechanical properties [Kodama et al., 2000; Notomi et al., 2001]. However, the improvement in mechanical properties cannot be fully explained by changes in the size and shape of bones, and mechanical loading can also affect the quality of the extracellular matrix (ECM) [Wallace et al., 2007]. The effects of exercise on skeletal fragility, and how alterations in fragility are modulated by changes in the composition of bone remain unknown. In this paper, we summarize a series of investigations in which the effects of exercise on the size, composition, mechanical properties and damage resistance of bones from mice of various ages, background strains and genetic makeup were assessed, as a means of testing the hypothesis that mechanical loading can improve mechanical properties of bone in the absence of changes in amount or distribution.

\section{Materials and Methods}

At 16 weeks of age, 60 adult male C57BL/6 mice were randomly assigned to weight-matched control or exercise groups. Exercise consisted of running on a treadmill at $12 \mathrm{~m} / \mathrm{min}$ on a $5^{\circ}$ incline, $30 \mathrm{~min} /$ day for 21 consecutive days [Wallace et al., 2007]. Mice were sacrificed on day 22 and tibiae were analyzed by microcomputed tomography to assess cross-sectional geometric properties of the tibial cortices. From each mouse, a randomly selected tibia $(\mathrm{n}=30$ ) was fatigued (4-point bending, $90 \mathrm{MPa}, 2 \mathrm{~Hz}, 21,600$ cycles), with the contralateral tibia used as a nonfatigued control. Half the tibiae in each group $(n=15)$ were designated for mechanical testing, while the other half was evaluated for microdamage. Structural level mechanical properties were obtained by testing bones to failure monotonically, while tissue level properties were estimated by calculating stresses and strains using the geometric properties obtained from microcomputed tomography and beam-bending theory. Specimens analyzed for microdamage

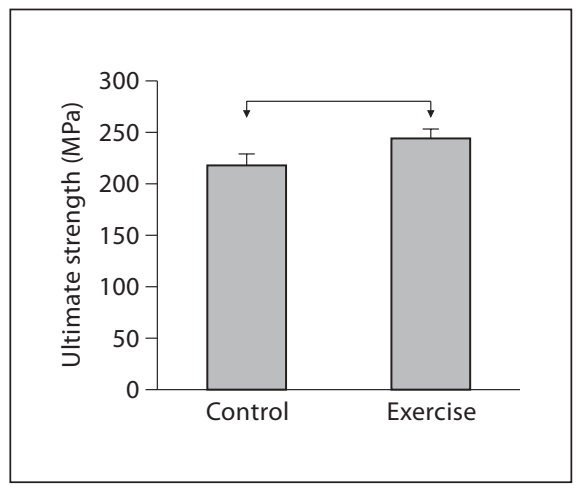

Fig. 1. The ultimate tensile strength of tibial cortical bone of 4-month-old C57BL/6 male mice subjected to short-term exercise is significantly greater than that of nonexercised controls $(\mathrm{p}<$ 0.05 ; denoted by horizontal bar between groups). Data are presented as means \pm SEM.

were bulk stained in basic fuchsin, embedded in PMMA, sectioned transversely through the mid-diaphysis, mounted and polished to $120 \mu \mathrm{m}$. Microdamage was quantified on a confocal microscope.

To assess tissue composition, Raman spectra were acquired using a Raman microprobe with a line-focused 785-nm near-infrared laser through a $20 \times / 0.75$ objective [Timlin et al., 2000; Carden et al., 2003]. Each tibia was examined at 4 points, endosteally to periosteally, in each of 4 quadrants, anterior, posterior, medial and lateral, and data were averaged. As measures of mineral/ matrix and carbonate/phosphate ratios, the band area ratios of phosphate $v_{1}\left(958 \mathrm{~cm}^{-1}\right) /[$ hydroxyproline + proline $(851+873+$ $\left.\left.917 \mathrm{~cm}^{-1}\right)\right]$ and carbonate $v_{1}\left(1,070 \mathrm{~cm}^{-1}\right) /$ phosphate $v_{1}\left(958 \mathrm{~cm}^{-1}\right)$ were used, respectively. The ratio of the band areas 1,660 to 1,690 $\mathrm{cm}^{-1}$ under the amide I envelope, termed cross-linking ratio, was used as a metric for the amount of nonreducible/reducible crosslinking in type I collagen [Paschalis et al., 2001].

Effects of exercise and fatigue on geometric and mechanical properties, microdamage and Raman band area ratios were made by 2-way ANOVAs. Similar protocols, except for fatigue loading and damage quantification, were used on 2-month-old biglycandeficient and wild-type mice bred on a C57BL/6x129 (B6;129) background.

\section{Results}

In 4-month-old C57BL/6 male mice, exercise led to a significant increase in ultimate strength (fig. 1), without significantly increasing cross-sectional size or shape (cortical area, cortical thickness, bending moment of inertia; data not shown), suggesting that mechanical stimulation led to changes to the preexisting bone matrix and that these changes in matrix quality were associated with improvements in mechanical properties. The min- 
Fig. 2. Ratios of number of microcracks (a) and reducible to irreducible cross-links (b) in fatigued bones relative to nonfatigued bones of control and exercised mice. ${ }^{*} \mathrm{Fa}$ tigue loading had a significant effect on the measured property. Changes in bone composition and damage accumulation brought about by fatigue loading diminished with exercise. With exercise, damage resistance, as indicated by both microcracking and collagen cross-linking, are improved.

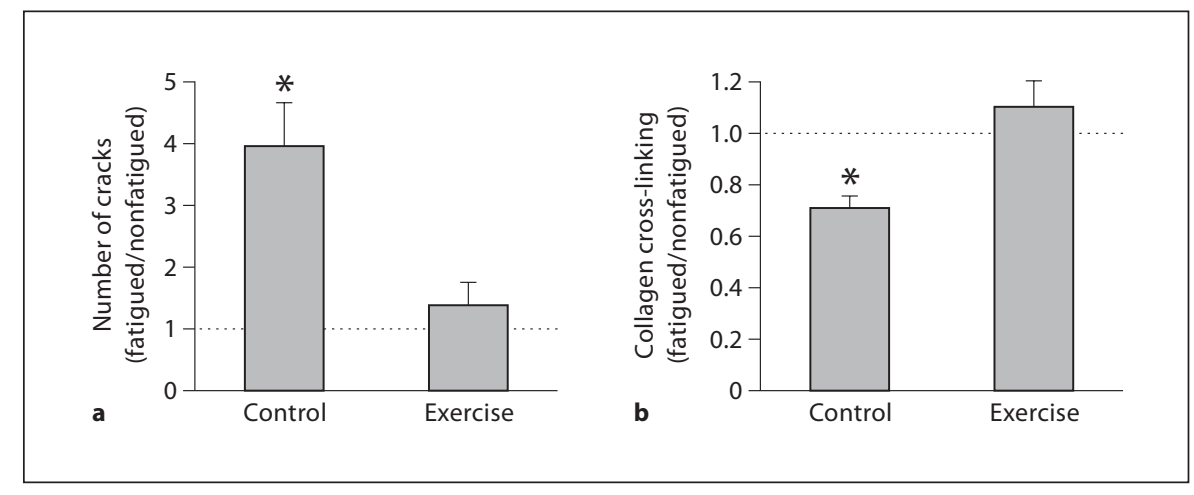

eral/matrix ratio was significantly increased in exercised bones relative to controls $(6.36 \pm 0.22$ vs. $5.52 \pm 0.24$; $\mathrm{p}<0.027)$ and the carbonate/phosphate ratio was significantly reduced $(0.193 \pm 0.044$ vs. $0.225 \pm 0.031 ; \mathrm{p}<$ 0.022 ). The increase in the relative amount of mineral and change in the nature of the mineral are hypothesized to be contributory factors in the exercise-induced increase in strength.

There were no significant differences in the number of microcracks, crack length or diffuse damage area between control and exercise groups before fatigue, indicating that the exercise regime did not compromise skeletal integrity. Fatigue loading of bones from nonexercised mice resulted in significant increases in the number of microcracks (fig. $2 \mathrm{a} ; \mathrm{p}<0.002$ ), crack length and diffuse damage area (data not shown). However, when bones from mice subjected to exercise were fatigued, there was no increase in any damage metric compared to nonfatigued bones (fig. 2a), indicating that exercise made the tibiae more resistant to fatigue-induced damage accumulation, and providing some of the first direct evidence that exercise modulates fracture resistance.

In bones from animals not subjected to exercise, fatigue loading led to a significant decrease in mineral/matrix ratio $(5.52 \pm 0.24$ vs. $4.70 \pm 0.24 ; \mathrm{p}<0.024)$. The $1,660 / 1,690$ band area ratio also decreased with fatigue (fig. 2b; $\mathrm{p}<0.003$ ), and is interpreted as a larger amount of disordered secondary structure in amide I, likely due to rupture of cross-links. The Raman data therefore show that there are significant changes in tissue quality associated with fatigue damage. Fatigue loading following exercise did not result in changes in mineral/matrix ratio $(6.36 \pm 0.24$ vs. $6.27 \pm 0.29 ; p<0.80)$ or cross-linking ratios (fig. 2b). Therefore, exercise prior to fatigue loading prevented the changes in composition that occurred when non-exercised bones were fatigued. Taken together, the Raman data parallel the microdamage data, albeit on a different scale, and show that exercise diminishes ultrastructural changes in the bone matrix brought about by fatigue.

As a second example of the ability of exercise to influence mechanical properties in the absence of cross-sectional geometric changes, type B carbonate levels $(0.090$ \pm 0.001 vs. $0.087 \pm 0.001 ; \mathrm{p}<0.036)$ and levels of mature collagen cross-links $(2.95 \pm 0.04$ vs. $2.85 \pm 0.04$; $p<$ $0.09)$ were elevated with exercise in biglycan-deficient mice. These compositional differences with exercise were associated with a marginally significant increase in yield strength $(206 \pm 9.0$ vs. $186 \pm 7.3 \mathrm{MPa} ; \mathrm{p}<0.09)$, but failed to induce an increase in bone formation, suggesting that exercise-induced compositional changes reflect alterations in preexisting tissue.

As a third example of the ability of exercise to influence mechanical properties without changes in bone size and shape, exercise led to a marginally significant increase in failure strain $(30,568 \pm 2,641$ vs. $24,254 \pm$ $1,791 \mu \varepsilon ; \mathrm{p}<0.06)$ and toughness $(4.47 \pm 0.41$ vs. 3.59 $\pm 0.31 \mathrm{MPa} ; \mathrm{p}<0.097)$ in 2 -month-old B6;129 mice. The marginal increase in collagen cross-linking ratio that also occurred with exercise $(3.19 \pm 0.04$ vs. $3.09 \pm 0.04$; $\mathrm{p}<0.098$ ) may be responsible for this change, as collagen alterations are associated with postyield behavior [Boskey et al., 1999; Wang et al., 2002].

\section{Discussion}

In mice from different ages, background strains and genetic makeup, exercise increases structural and tissue level mechanical properties compared with weightmatched control mice, without changes in bone size or shape. Coupled with the absence of changes in bone for- 
mation rate and lack of intracortical remodeling in mice, these results suggest that mechanically induced changes in preexisting bone quality are responsible for the increases in mechanical properties. Moreover, tissue level properties changed with exercise, and properties at this level of hierarchy are independent of bone size, providing further confirmation that these effects were driven by changes in the quality of the preexisting ECM.

It is commonly stated that the primary effect of mechanical stimulation is an increase in bone mass [Carter et al., 1996]. However, mechanical loading also has effects on bone that influence the quality of the ECM [Kohn et al., 2005; Wallace et al., 2007]. Since many diseases influence the mechanical integrity of bone via alterations in tissue quality, our results suggest that mechanical stimulation may be a practical way to prevent and treat ECM deficiencies.

A consistent result across mice from different ages, background strains and genetic deficiencies was that exercise led to changes in the degree of mineralization and/ or mineral composition. However, the specific compositional changes brought about by mechanical stimulation were dependent on age, background strain and genotype. While the influence of mechanical loading on bone mineral density has been demonstrated [Kesavan et al., 2005; Wallace et al., 2007], this is the first indication of changes in the nature of mineral with loading.

The mechanisms driving mechanically mediated changes in mineral are unknown, and may be physicallychemically and/or biologically mediated. In synthetic ceramics, pressure-induced phase changes and/or substitutions can occur [Vaidya et al., 1997]. In bone, changes in Raman spectra at crack tips have been interpreted as transformations [Timlin et al., 2000]. Other potential mechanisms can be related to the supersaturated microenvironment and increase in fluid flow with mechanical loading. Exercise increases intestinal absorption of $\mathrm{Ca}^{2+}$, $\mathrm{PO}_{4}^{2-}$ and $\mathrm{CO}_{3}^{2-}$, increasing serum concentrations of these ions [Yeh et al., 1989]. Exercise also increases fluid flow through bone [Duncan and Turner, 1995; Burger and Klein-Nulend, 1999]. Increased mineral ion concentrations coupled with increased fluid flow near crystallites having a large surface/volume ratio [Weiner and Traub, 1992; Landis, 1996] could lead to changes in mineral chemistry via substitutions or crystal size. Other potential mechanisms include changes in the interface between mineral and collagen, affecting load transfer and/or residual stresses [Gupta et al., 2006; Almer and Stock, 2007], or changes in structural water that exists at the interface between mineral and collagen that can affect mechanical properties of the bone composite [Wilson et al., 2005, 2006]. The existence of physically-chemically mediated mechanisms of compositional changes in bone does not rule out underlying cellular mechanisms, and the 2 are not mutually exclusive. In fact, mRNA levels of collagen and several noncollagenous proteins (fibromodulin, osteopontin, biglycan), along with TGF- $\beta$ and alkaline phosphatase were significantly elevated in exercised bones, compared to controls [Wallace, 2007].

In summary, a short-term exercise regime that does not lead to an increased amount of bone can still decrease skeletal fragility via alterations in the existing bone matrix. Coupling mechanical loading with Raman spectroscopy has identified specific constituents in bone ultrastructure that are associated with alterations in mechanical properties and damage accumulation. This study therefore demonstrates the importance of considering composition, and not just bone mass, in diagnosing and evaluating skeletal fragility, and also demonstrates the potential for exogenous mechanical stimulation to affect mechanical integrity via changes in bone quality and not quantity.

\section{Acknowledgements}

This work was supported by DoD/US Army DAMD17-03-10556, NIH R01 AR 052010 and NIH R90-DK071506.
References
Kohn/Sahar/Wallace/Golcuk/Morris 
-Carter, D.R., M.C. Van Der Meulen, G.S. Beaupre (1996) Mechanical factors in bone growth and development. Bone 18: 5S-10S.

Duncan, R.L., C.H. Turner (1995) Mechanotransduction and the functional response of bone to mechanical strain. Calcif Tissue Int 57: 344-358.

Gupta, H.S., J. Seto, W. Eagermaier, P. Zaslansky, P. Boesecke, P. Fratzl (2006) Cooperative deformation of mineral and collagen in bone at the nanoscale. Proc Natl Acad Sci USA 103: 17741-17746.

-Kesavan, C., S. Mohan, S. Oberholtzer, J.E. Wergedal, D.J. Baylink (2005) Mechanical loading-induced gene expression and BMD changes are different in two inbred mouse strains. J Appl Physiol 99: 1951-1957.

Kodama, Y., Y. Umemura, S. Nagasawa, W.G. Beamer, L.R. Donahue, C.R. Rosen, D.J. Baylink, J.R. Farley (2000) Exercise and mechanical loading increase periosteal bone formation and whole bone strength in C57BL/6J mice but not in $\mathrm{C} 3 \mathrm{H} / \mathrm{Hej}$ mice. Calcif Tissue Int 66: 298-306.

Kohn, D.H., N.D. Sahar, S.I. Hong, K. Golcuk, M.D. Morris (2005) Local mineral and matrix changes associated with bone adaptation and microdamage; in Bushby, A.J., V.L. Ferguson, C.C. Ko, M.L. Oyen (eds): Mechanical Behavior of Biological and Biomimetic Systems. Mater Res Soc Symp Proc, Warrendale, Materials Research Society, vol 898E, paper 0898-L09-03.
Landis, W.J. (1996) Mineral characterization in calcifying tissues: atomic, molecular and macromolecular perspectives. Connect Tissue Res 34: 239-246.

Notomi, T., N. Okimoto, Y. Okazaki, Y. Tanaka, T. Nakamura, M. Suzuki (2001) Effects of tower climbing exercise on bone mass, strength, and turnover in growing rats. J Bone Miner Res 16: 166-174.

Paschalis, E.P., K. Verdelis, S.B. Doty, A.L. Boskey, R. Mendelsohn, M. Yamauchi (2001) Spectroscopic characterization of collagen cross-links in bone. J Bone Miner Res 16: 1821-1828.

Timlin, J.A., A. Carden, M.D. Morris, R.M. Rajachar, D.H. Kohn (2000) Raman spectroscopic imaging markers for fatigue-related microdamage in bovine bone. Anal Chem 72: 2229-2236.

United States Department of Health and Human Services, Office of the Surgeon General (2004) Bone Health and Osteoporosis: A Report of the Surgeon General. La Vergne, Lightning Source.

Vaidya, S., C. Karunakaran, B. Pande, N. Gupta, R. Iyer, S. Karweer (1997) Pressure-induced crystalline to amorphous transition in hydroxylapatite. J Mater Sci 32: 3213-3217.
Wallace, J.M. (2007) Investigating the Inbred Strain-Specific Response to Biglycan-Deficiency and Exercise: A Study in GeneticallyMedicated Skeletal Adaptation; thesis, University of Michigan.

Wallace, J.M., R.M. Rajachar, M.R. Allen, S.A. Bloomfield, P.G. Robey, M.F. Young, D.H. Kohn (2007) Exercise-induced changes in the cortical bone of growing mice are boneand gender-specific. Bone 40: 1120-1127.

Wang X., X. Shen, X. Li, C.M. Agrawal (2002) Age-related changes in the collagen network and toughness of bone. Bone 31:1-7.

Weiner, S., W. Traub (1992) Bone structure: from angstroms to microns. FASEB J 6: 879-885.

Wilson, E.E., A. Awonusi, M.D. Morris, D.H. Kohn, M.M.J. Tecklenburg, L.W. Beck (2005) Highly ordered interstitial water observed in bone by nuclear magnetic resonance. J Bone Miner Res 20: 625-634.

-Wilson, E.E., A. Awonusi, M.D. Morris, D.H Kohn, M.M.J. Tecklenburg, L.W. Beck (2006) Three structural roles for water in bone observed by solid state NMR. Biophys J 90: 3722-3731.

Yeh, J.K., J.F. Aloia, S. Yasumura (1989) Effect of physical activity on calcium and phosphorus metabolism in the rat. Am J Physiol 256: E1E6. 